\title{
VPS35 Protects Against TMEM230-Mutation- Induced Progressive Locomotor Deficits in Drosophila
}

\section{Chao Ma}

Tsinghua University School of Medicine https://orcid.org/0000-0002-6514-1707

\section{Xiaobo Wang}

Johns Hopkins University School of Medicine

Wanli W Smith

Johns Hopkins University School of Medicine

Zhaohui Liu ( $\square$ zhliu@suda.edu.cn )

https://orcid.org/0000-0003-3106-9206

\section{Research article}

Keywords: Parkinson's disease, TMEM230, Mitogen-activated protein kinase, Vacuolar protein sorting-35, JNK, caspase-3

Posted Date: October 26th, 2021

DOl: https://doi.org/10.21203/rs.3.rs-952504/v1

License: (c) (i) This work is licensed under a Creative Commons Attribution 4.0 International License. Read Full License 


\section{Abstract}

\section{Background}

Recently, four Parkinson's disease (PD)-linked mutations (Y92C, R141L, 184PGext*5 and 184Wext*5) in transmembrane protein 230 (TMEM230) were identified in PD patients, and these mutations have implications in protein trafficking and neurodegeneration. However, there is a lack of in vivo studies on the roles of PD-related variants of TMEM230 in PD pathogenesis.

Methods

In this study, we generated human wild-type (WT) and mutant TMEM230 (Y92C, R141L, 184PGext*5 and 184 Wext ${ }^{\star 5}$ ) transgenic Drosophila using isoform $\otimes$ cDNA.

Results

We found that the expression of TMEM230 184PGext $* 5$ in pan-neurons or dopaminergic neurons in Drosophila induced PD-like phenotypes, which included impaired locomotor ability, a shortened lifespan, reduced TH levels, and increased phosphorylated JNK and cleaved caspase-3 levels. Moreover, rotenone, a common pesticide, enhanced TMEM230-184PGext ${ }^{*}$-induced PD-like phenotypes. In contrast, the overexpression of wild-type (WT) VPS35 rescued TMEM230-184PGext 5 -induced PD-like phenotypes, while the knockdown of VPS35 by RNA interference (RNAi) or the expression of mutant VPS35 D620N worsened PD-like phenotypes.

Conclusion

These results indicate that VPS35, as a downstream effector of TMEM230, plays a critical role in TMEM230-linked JNK/caspase-3 signalling pathways and that mutations in TMEM230 and VPS35 disrupt these pathways, resulting in dopaminergic neurodegeneration and PD-like phenotypes. These findings provide novel insight into the molecular mechanisms of mutant TME230- and VPS35-induced abnormalities underlying the pathogenesis of PD.

\section{Background}

Parkinson's disease (PD) is the second most common neurodegenerative disease, with classic motor features of resting tremor, rigidity, bradykinesia and postural instability. Selective loss of dopaminergic neurons (DNs) in the substantia nigra pars compacta (SN) and the presence of Lewy bodies that include insoluble a-synuclein aggregates are hallmarks of PD pathology. A total of $5-10 \%$ of PD patients suffer from a monogenic form of the disease with Mendelian inheritance. However, the underlying mechanism of PD pathogenesis remains largely unknown[1].

Recently, a new PD-related gene, transmembrane protein 230 (TMEM230), was identified in PD patients [2]. Four PD-linked TMEM230 mutations were identified [2], including Y92C, R141L, 184PGext*5 (replacing 
the stop codon with proline and glycine, thus adding seven amino acids, PGHPPHS, to the C-terminus) and $184 \mathrm{Wext} * 5$ (replacing the stop codon with six amino acids, WHPPHS). Y92C, R141L and $184 \mathrm{Wext} * 5$ are found in North American patients with PD, whereas the 184PGext ${ }^{\star} 5$ mutation is identified in Chinese families with PD [2]. Subsequently, studies have suggested that TMEM230 variants are related to PD pathogenesis in vitro [3-5]. However, there is a lack of in vivo studies on the roles of PD-related variants of TMEM230 in PD pathogenesis.

The TMEM230 gene encodes two protein isoforms [2], the 183-amino-acid isoform- 1 and the 120 -aminoacid isoform-2. Isoform-2 accounts for more than 95\% of the TMEM230 protein isoforms in humans (Figure 1A). The physiological and pathological cellular functions of TMEM230 in PD are still elusive [6]. Increasing evidence suggests that TMEM230 may be involved in various cellular functions, such as vesicle trafficking [2, 3], trans-Golgi network secretion [2, 3], Rab family proteins in the endosomal pathway $[2,3,7]$, cell toxicity $[4,5,7]$, protein aggregation and the autophagy lysosomal pathway $[2,3,7]$. Studies suggest that mutant TMEM230 may alter these cellular pathways and has implications in PD and other related disorders.

VPS35 protein is a major component of the retromer complex involved in endosome-to-Golgi retrieval and membrane-protein recycling [8]. The autosomal-dominant mutation D620N in VPS35 has been identified in PD patients $[9,10]$. Studies have demonstrated that the VPS35 D620N mutation disrupts the association of VPS35 with other trafficking proteins, leading to protein trafficking defects $[11,12]$. TMEM230 has been shown to colocalize with VPS35 and the retromer complex [2]. TMEM230 R141L and $184 W_{\text {ext }}^{\star} 5$ variants disrupt VPS35 cytoplasmic distribution in a cell[3]. However, whether there is physiological interplay between VPS35 and TMEM230 in vivo is still unclear.

In this study, we generated wild-type (WT) and mutant TMEM230 transgenic Drosophila to study the roles of TMEM230 and its interplay with VPS35 using genetic tools. Our studies provide novel insight into the roles of TMEM230 and its interaction with rotenone (Rot) and VPS35 in PD pathogenesis.

\section{Materials And Methods}

\section{Drosophila genetics}

All Drosophila stocks were maintained with a 12 hours light/dark cycle at $25^{\circ} \mathrm{C}$ and $55 \%$ humidity, raised in non-crowded conditions on standard cornmeal medium. Fly food consisted of (per $1 \mathrm{~L}$ ) $10 \mathrm{~g}$ agar, 7.25 $\mathrm{g}$ sucrose, $30 \mathrm{~g}$ glucose, $24.5 \mathrm{~g}$ yeast, $50 \mathrm{~g}$ corn meal, $17.5 \mathrm{ml}$ methyl 4-hydroxybenzoate, $4 \mathrm{ml}$ propionic acid. The control flies used for this study were W1118 strain. We generated UAS-TMEM230-WT, UASTMEM230-Y92C, $-R 141 L,-184 W e x{ }^{\star} 5$ and $-184 P G e x t \star 5$ transgenic flies as described previously[13]. Briefly, UAS-TMEM230-WT, UAS-TMEM230-Y92C, -R141L, - 184Wext ${ }^{\star} 5$ and $-184 P G e x t^{\star} 5$ is oform $\llbracket \mathrm{CDNA}$ werecloned between EcoRI and Kpnl sites of pUAST vector (Figure 1A) and verified by sequencing. The resulting constructs were microinjected in $w^{1118}$ fly embryos. TH-GAL4 and elav-GAL4 were obtained fromBloomington Drosophila Stock Center (BDSC), UAS-VPS RNAi was obtained from Vienna Drosophila 
Resource Center (VDRC), UAS-VPS35, and UAS-VPS35-D620N were generated by our lab. The following genetic combinations were used to express transgenes in adult neurons: (1) elav-GAL4/+; UAS-TMEM230WT/+, (2) elav-GAL4/+; UAS-TMEM230-Y92C/+, (3) elav-GAL4/+;UAS-TMEM230-R141L/+, (4) elavGAL4/+; UAS-TMEM230-184PGext*5/+, (5) elav-GAL4/+; UAS-TMEM230-184Wext*5/+. The following genetic combinations were used to express transgenes in adult dopaminergic neurons: (1) THGAL4/+;UAS-TMEM230-WT/+,(2) TH-GAL4/+;UAS-TMEM230-184PGext*5/+, (3) TH-GAL4/+;UAS-VPS35-

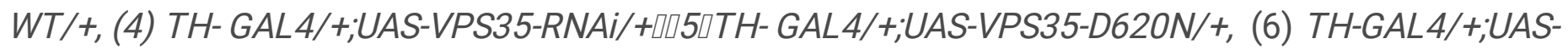
VPS35-WT/+;UAS-TMEM230-184PGext*5/+,(7) TH-GAL4/+;UAS-VPS35-RNAi/+;UAS-TMEM230184PGext ${ }^{\star} 5 /+, \varangle 8 \otimes T H$ GAL4/+,UAS-VPS35-D620N/+;UAS-TMEM230-184PGext*5/+.

\section{Western blot analysis}

Adult fly heads were homogenized using RIPA buffer $(50 \mathrm{mM}$ Tris- $\mathrm{HCl}, \mathrm{pH} 8.0,150 \mathrm{mM} \mathrm{NaCl}, 0.25 \%$ sodium deoxycholate, $0.1 \%$ SDS, $1 \%$ NP-40, supplemented with complete protease inhibitor mixture; Roche). The homogenates were boiled with SDS loading buffer, were separated on SDS-PAGE gels and were transferred to PVDF membranes (GE Healthcare). Blots were incubated with the primary antibodies overnight at $4^{\circ} \mathrm{C}$, and then HRP-conjugated secondary antibodies at room temperature for $1 \mathrm{~h}$. Primary antibodies were used including anti-tyrosine hydroxylase (TH)( ab112, Abcam Inc; 1:1000), anti-phosphoERK (Thr202/Tyr204), anti-ERK, anti-phospho-SAPK/JNK (Thr183/Tyr185) or anti-JNK (1:2000,Cell Signaling Technology), anti-cleaved human caspase 3 (1:2000, Cell Signaling Technology), and mouse anti- $\beta$-tubulin antibody $(1: 5,000)$.

\section{Fly climbing assay}

Adult male flies were put into a $50 \mathrm{~mL}$ measuring cylinder. Cohorts of 60 flies from each genotype were subjected to assay every 10 days from 5 days after eclosion. The flies were gently tapped to the bottom of a vial and allowed to climb for 30 seconds. The climbing ability of flies was quantified as number of animals that reach the $10 \mathrm{~cm}$ of cylinder in 15 seconds. The test was repeated 3 times for each group. The number of flies that reached the $10 \mathrm{~cm}$ was converted into a percentage value, the Turkey's test after one-way analysis of variance (One-way ANOVA) was used for data analysis.

\section{Flight assay}

The flight assay was performed as described in [14]. Briefly, adult flies at age of 5,15, or 25 days, were subjected to flight assays. Flies were anaesthetized on ice for 5 minutes, then a thin metal wire was glued between the neck and thorax region with the assist of nail polish. After allowing them to recover for $\sim 5-7$ minutes, a gentle mouth-blown air puff then was given and flight time was recorded using a stop watch. Flight duration was recorded for a maximum of fifteen minutes for each fly in batches of 5-8 flies at a time. For each genotype, each experimental group, a minimum of 20 flies were tested. 


\section{Life span assay}

Adult male flies (60 flies per group) from each group were used for survival analyses. Flies were grown at $25^{\circ} \mathrm{C}$ and moved to fresh medium every 4 days. Death was recorded every other day until all flies from the experimental groups were dead. Log-rank tests were performed for statistical analysis via Prism GraphPad software.

\section{Rotenone exposure method}

The flies were exposed to varying concentrations $(0.25 \mathrm{mM}, 0.5 \mathrm{mM}$ and $0.75 \mathrm{M})$ of rotenone (Rot) for 7 days in order to assess the lethality responses to choose the effective dose for LD $_{50}$ [15]. After segregation of 20 flies per vial into four groups (three vials in each group). Group I control flies fed with cornmeal medium. Group II to IV flies received ingestion of rotenone $(0.25 \mathrm{mM}, 0.5 \mathrm{mM}$ and $0.75 \mathrm{M})$ through the medium for seven consecutive days. On the end of experiment ( $8^{\text {th }}$ day), flight assay and western blot analysis were performed as described above.

\section{Results}

Expression of TMEM230 184PGext ${ }^{\star}$ and 184 Wext ${ }^{\star} 5$ in pan-neurons induces locomotor deficits and shortens the fly lifespan

To study the role of TMEM230 in neurodegenerative pathology in the fly model, we generated human WT and mutant TMEM230 (Y92C, R141L, 184Wext*5 and 184PGext*5) transgenic flies using TMEM230 isoform-2 cDNA as described previously[5]. We observed the lifespan of elav-GAL4; TMEM230 flies (WT, $Y 92 C, R 141 L, 184 P G e x t^{\star} 5$ and 184 Wext $\left.* 5\right)$, in which the TMEM230 protein is expressed in all neurons. We found that elav-GAL4;184Wext*5 and elav-GAL4;184PGext ${ }^{\star} 5$ flies had significantly shortened lifespans compared with non-transgenic normal control flies (Fig. 1C). The lifespan of elav-GAL4; Y92C and elav-GAL4; R141L flies was slightly decreased (but not significantly) compared with that of elavGAL4; TMEM230 flies. (Figure 1C).

Flight and climbing assays are often used to assess motor functions in flies, as described previously [13, 14]. The climbing and flight abilities of WT and mutant TMEM230 flies were evaluated at time points after eclosion (climbing days: $5,15,25,35,45,55$; flight days: $5,15,25$ ). We found that elavGAL4;184PGext ${ }^{*}$ and elav-GAL4;184Wext*5 flies displayed age-related locomotor impairment, while elav-GAL4; Y92C and elav-GAL4; R141L flies exhibited slightly decreased climbing activities but no significant difference compared with non-transgenic flies (Figure 1D). Similarly, the flight ability of elavGAL4;184PGext*5 and elav-GAL4;184Wext*5 flies was significantly impaired, while the flight ability of elav-GAL4; Y92C and elav-GAL4; R141L flies was only slightly impaired compared with that of nontransgenic flies (Figure 1B). These results suggest that the TMEM230 184PGext ${ }^{\star 5}$ and 184 Wext ${ }^{\star 5}$ mutations induced more severe PD-like behavioural impairment in the fly models. Since elavGAL4;184PGext*5 was more neurotoxic than elav-GAL4;184Wext*5 in flies (Figure 1), we then prioritized 
our investigation of the $184 P G e x t \star 5$ mutation in this study; thus, only TMEM230 WT and $184 P G e x t^{\star} 5$ data are reported in the rest of this study.

\section{Expression of TMEM230 184PGext*5 in DNs evokes locomotor deficits}

Given that PD is characterized by a loss of DNs in the brain [16], we expressed WT and mutant TMEM230 in DNs using TH driver flies. We found that TH-GAL4;184PGext ${ }^{\star} 5$ flies displayed a shorter lifespan than TH-GAL4; TMEM230 flies (Fig. 2A). Homozygous TH-GAL4;184PGext*5 flies died earlier than heterozygous TH-GAL4;184PGext ${ }^{\star} 5$ flies (Figure 2A). Moreover, TH-GAL4;184PGext ${ }^{\star} 5$ flies showed locomotor deficits that were comparable with those of TH-GAL4; TMEM230 flies (Figure 2C). Homozygous TH-GAL4;184PGext*5 flies had more severe locomotor impairment than heterozygous THGAL4;184PGext*5 flies (Figure 2C). Similarly, TH-GAL4;184PGext ${ }^{*}$ flies showed flight ability impairment that was comparable with that of TH-GAL4; TMEM230 flies (Figure 2B).

Tyrosine hydroxylase (TH), a marker of DNs [17], is often used to test the lesion and/or loss of DNs in THGAL4;184PGext ${ }^{\star}$ flies. There was a significant decrease in TH protein levels in TH-GAL4;184PGext*5 flies compared with TH-GAL4; TMEM230 flies (Figure 2D). Homozygous TH-GAL4;184PGext 5 flies had lower TH protein levels than heterozygous TH-GAL4;184PGext*5 flies (Figure 2D). These results demonstrated that TMEM230 184PGext ${ }^{\star 5}$ induced DN degeneration in an age- and dose-dependent manner.

\section{PGext*5 accelerates Rot-induced neurotoxicity in flies}

Rot, a mitochondrial complex I inhibitor, is often used to induce oxidative damage and dopaminergic neurodegeneration to model parkinsonism in Drosophila models [18]. Consistent with our previous studies, flies exposed to $0.75 \mathrm{mM}$ Rot for 8 days showed increased mortality (up to $50 \%$ ) compared with flies not exposed to Rot (Figure 3A). Rot-exposed flies also showed decreased TH protein levels and flight abilities compared with non-Rot-exposed flies (Figure 3B and C). Most interestingly, only $\mathrm{TH}$ GAL4;184PGext 5 flies exposed to $0.25 \mathrm{mM}$ Rot showed significantly increased mortality compared with TH-GAL4; TMEM230 flies (Fig. 3D). In addition, TH-GAL4;184PGext*5 flies exposed to $0.50 \mathrm{mM}$ Rot showed significantly exacerbated locomotor impairment compared with TH-GAL4; TMEM230 flies exposed to $0.50 \mathrm{mM}$ Rot; however, there was no difference in locomotor impairment between these two transgenic fly groups in the absence of Rot exposure (Figure 3E). These results demonstrate that the TMEM230 184PGext ${ }^{\star}$ mutation leads to increased sensitivity to Rot.

\section{TMEM230 184PGext`5 increases JNK phosphorylation and caspase-3 activation in flies}

Oxidative stress plays a major role in mediating DN pathology [19], in which the c-Jun N-terminal kinase (JNK) pathway is activated and subsequently promotes inflammation and cell death [20,21]. A previous study demonstrated that $184 W_{\text {est }}^{*}$ and 184 PGext* increase reactive oxygen species (ROS) levels in mitochondria and induce caspase $3 / 7$ activation and poly (ADP-ribose) polymerase (PARP) cleavage in vitro [5]. We were interested in studying whether the TMEM230 184PGext*5 mutation alters the JNK 
pathway and caspase-3 activation. We found that TH-GAL4;184PGext ${ }^{\star} 5$ flies showed significantly increased JNK (p-JNK) phosphorylation (Figure 4A-B) and increased cleaved caspase-3 levels (Figure 4C) compared with non-transgenic and WT-TMEM230 flies.

\section{Expression of VPS35 reduces TMEM230-184PGext*5-induced JNK phosphorylation and caspase-3 cleavage in flies}

Retromer dysfunction plays a critical role in neurodegeneration in some PD models [22]. VPS35 is the core of the retromer complex, and mutations in VPS35 disrupt protein accumulation and clearance and have implications in PD [22]. Our results showed that VPS35 expression decreased p-JNK protein levels in TH-GAL4; VPS35:184PGext ${ }^{*}$ flies (Fig. 5A and 5B). In contrast, the knockdown of VPS35 by RNA interference (RNAi) further elevated 184PGext*5-induced JNK phosphorylation in TH-GAL4; VPS35RNAi:184PGext ${ }^{\star} 5$ flies compared with TH-GAL4;184PGext ${ }^{\star} 5$ flies (Figure 5B). Moreover, VPS35 decreased $184 P G$ ext ${ }^{\star} 5$-induced Caspase- 3 cleavage in TH-GAL4; VPS35:184PGext $\star 5$ flies compared with that in TH-GAL4;184PGext*5 flies (Figure 5C).

\section{VPS35 alleviates TMEM230-184PGext`5-induced PD-like phenotypes in flies}

The overexpression of VPS35 in DNs by TH driver flies increased TH protein levels (Figure 6A), increased flight duration time (Figure 6B), enhanced locomotor ability (Figure 6C) and extended longevity (Figure 6D) in TH-GAL4; VPS35:184PGext ${ }^{*} 5$ flies compared with TH-GAL4;184PGext*5 flies. Previous studies showed that the PD-like mutation VPS35-D620N, a point mutation, disrupts VPS35 function[11]. We found that the overexpression of VPS35-D620N worsened the PD-like phenotypes in TH-GAL4; VPS35D620N:184PGext ${ }^{*}$ flies compared with TH-GAL4;184PGext*5 flies (Figure 6). These results suggest that VPS35 may act as a downstream effector of TMEM230 and play an important role in dopaminergic neurodegeneration and PD phenotypes.

\section{Discussion}

In this study, we generated human WT and mutant TMEM230 (Y92C, R141L, 184PGext*5 and 184 Wext ${ }^{\star}$ ) transgenic Drosophila. We found that the expression of either TMEM230 184PGext ${ }^{\star}$ or 184 Wext ${ }^{\star} 5$ in pan-neurons or in DNs in Drosophila induced PD-like phenotypes, which included impaired locomotor ability, a shortened lifespan, reduced TH levels, and increased phosphorylated JNK and cleaved caspase-3 levels. TMEM230 184PGext $\star 5$ caused more severe PD-like phenotypes than other mutations. Moreover, Rot exposure enhanced TMEM230-184PGext ${ }^{\star 5}$-induced PD-like phenotypes. In contrast, the overexpression of WT VPS35 rescued TMEM230-184PGext*5-induced PD-like phenotypes, while the knockdown of VPS35 by RNAi or the expression of mutant VPS35 D620N worsened PD-like phenotypes. These results indicate that VPS35, as a downstream effector of TMEM230, plays a critical role in TMEM230-linked JNK/caspase-3 signalling pathways and that mutations in TMEM230 and VPS35 disrupt these pathways, resulting in dopaminergic neurodegeneration and PD-like phenotypes. 
Dopaminergic neurodegeneration is a key hallmark of PD pathology. We and others previously found that the expression of PD-linked TMEM230 variants induces cell death in vitro[5, 7]. In this study, we found that the expression of mutant TMEM230 184PGext ${ }^{\star} 5$ or $184 W$ ext ${ }^{\star} 5$ in pan-neurons or DNs decreased TH levels, impaired locomotor activation and shortened the lifespan in flies compared with WT TMEM230 or non-transgenic flies, which resembled the key features of human PD. However, flies expressing the TMEM230 variant ( $\mathrm{Y} 92 \mathrm{C}$ or $\mathrm{R} 141 \mathrm{~L}$ ) showed no changes compared with non-transgenic flies. This is consistent with the in vitro finding that TMEM230 184PGext*5 or 184 Wext ${ }^{\star} 5$ caused more severe neurotoxicity than the TMEM230 Y92C or R141L variants. Our studies provide the first in vivo TMEM230based PD Drosophila model for further pathogenesis studies.

In this study, we first found that TMEM230 184PGext ${ }^{\star} 5$ and $184 W e x{ }^{\star} 5$, but not $W T, Y 92 C$ and $R 141 L$, shortened longevity and reduced locomotive ability in flies, in which $184 P G e x t^{\star} 5$ induced more neurotoxicity than 184 Wext ${ }^{\star}$. We thus expressed $184 P G e x t^{\star} 5$ in DNs and found decreased locomotive abilities and lifespan and reduced TH protein levels in TMEM230 184PGext*5 DNs.

Oxidative damage caused by an increase in ROS suggests that ROS play a critical role in DN degeneration $[23,24]$. An increase in oxidative stress activates the JNK-MAPK signalling pathway and induces apoptosis $[25,26]$. Our results showed that exposure to Rot, which increased oxidative damage, enhanced mutant TMEM230-184PGext*5-induced PD-like phenotypes, suggesting that oxidative stress converges with genetic mutations in TMEM230-linked PD pathology.

Previously, we found that the expression of WT TMEM230 and the variants 184 Wext ${ }^{\star} 5$ and $184 P G$ ext ${ }^{\star} 5$ increased mitochondrial ROS levels, activated caspase 3/7 and induced PARP1 cleavage in cultured cells [5]. Consistent with in vitro studies, we found that p-JNK-MAPK and cleaved Caspase-3 were increased in TMEM230 $184 P$ Gext ${ }^{\star} 5$ flies. Moreover, the expression of WT VPS35 suppressed TMEM230-184PGext*5induced dopaminergic neurodegeneration and reduced JNK activation and caspase-3 cleavage. These results further demonstrated that the JNK/caspase-3 pathway mediates TMEM230-184PGext 5 -linked neurodegeneration and PD-like phenotypes.

TMEM230 colocalizes with VPS35 in synaptic vesicles (SVs), which is related to vesicle trafficking in trans-Golgi network secretion and has implications for Rab family proteins in the endosomal pathway, protein aggregation and the autophagy lysosomal pathway. The VPS35 D620N mutation has been identified in late-onset PD patients $[9,10]$. Previous studies showed that mutant VPS35 interacts with the retromer cargo and disrupts cellular trafficking [22]. Our results showed that VPS35 suppressed mutant TMEM230-induced PD-like phenotypes in flies. In contrast, the expression of mutant VPS35 D620N had no effect on mutant TMEM230-induced PD-like phenotypes. These findings indicated that VPS35 acts as a downstream effector and interacts with TMEM230. Mutations in either VPS35 or TMEM230 may disrupt this interplay, leading to neurodegeneration and PD-like phenotypes.

\section{Conclusions}


Cumulatively, we generated the first TMEM230-based PD Drosophila model in which the expression of human TMEM230 184 PGext ${ }^{\star} 5$ or $184 W_{\text {ext }}^{*} 5$ in pan-neurons or DNs induced parkinsonism-like behaviours. 184PGext ${ }^{\star} 5$ activated the JNK/caspase-3 pathway and increased dopaminergic neurodegeneration, while VPS35 attenuated 184 PGext*5-PD-like phenotypes by reducing JNK activation and caspase-3 cleavage. These findings provide novel insight into the mechanisms underlying mutantTMEM230- or mutant-VPS35-linked PD-like pathology. This TMEM230-based PD Drosophila model may provide a platform for further study of PD pathogenesis and therapeutics.

\section{Abbreviations}

PD: Parkinson's disease

TMEM230: transmembrane protein 230

VPS35: Vacuolar protein sorting-35

WT: wild-type

RNAi: RNA interference

DNs: dopaminergic neurons

SN: substantia nigra pars compacta

Rot: rotenone

TH: Tyrosine hydroxylase

JNK: c-Jun N-terminal kinase

ROS: reactive oxygen species

PARP: poly (ADP-ribose) polymerase

SVs: synaptic vesicles

\section{Declarations}

\section{Ethics declarations}

\section{Ethics approval and consent to participate}

This study was approved by the Ethics Committee of Soochow University. 


\section{Consent for publication}

All authors have given their consent for publication.

\section{Availability of data and materials}

Please contact the corresponding author for all data requests.

\section{Competing interests}

The authors declare that no competing interests exist.

\section{Funding}

This work was generously supported by grants from National Natural Science Foundation of China (grant No. 81371392) and the Jiangsu Natural Science Foundation (grant No. BK20131161). This project was also funded by the Priority Academic Program Development of Jiangsu Higher Education Institutions.

\section{Authors' contributions}

Data curation and Methodology, C.M.; Data analysis and Software, X.W.; Project administration, Z.L and W.W.S.; Funding acquisition and Supervision, Z.L.; Writing original draft, C.M. and Z.L.; Writing-review \& editing, W.W.S. All authors have read and agreed to the published version of the manuscript.

\section{Acknowledgements}

Not applicable.

\section{Authors' information}

\section{Affiliations}

Department of Human Anatomy and Cytoneurobiology, School of Biology and Basic Medical Sciences, Soochow University, Suzhou, Jiangsu 215123, China

Chao Ma, Xiaobo Wang, Zhaohui Liu.

Department of Psychiatry and Behavioral Sciences, Johns Hopkins University School of Medicine, Baltimore, MD 21287, USA

Xiaobo Wang, Wanli W. Smith. 


\section{Corresponding authors}

Correspondence to Wanli W. Smith and Zhaohui Liu.

\section{References}

1. Kouli A, Torsney KM, Kuan WL: Parkinson's Disease: Etiology, Neuropathology, and Pathogenesis. In: Parkinson's Disease: Pathogenesis and Clinical Aspects. edn. Edited by Stoker TB, Greenland JC. Brisbane (AU): Codon Publications Copyright: The Authors.; 2018.

2. Deng HX, Shi Y, Yang Y, Ahmeti KB, Miller N, Huang C, Cheng L, Zhai H, Deng S, Nuytemans K et al: Identification of TMEM230 mutations in familial Parkinson's disease. Nature genetics 2016, 48(7):733739.

3. Kim MJ, Deng HX, Wong YC, Siddique T, Krainc D: The Parkinson's disease-linked protein TMEM230 is required for Rab8a-mediated secretory vesicle trafficking and retromer trafficking. Human molecular genetics 2017, 26(4):729-741.

4. Wang X, Guo G, Zhang J, Aebez N, Liu Z, Liu CF, Ross CA, Smith WW: Mutant-TMEM230-induced neurodegeneration and impaired axonal mitochondrial transport. Human molecular genetics 2021, 30(16):1535-1542.

5. Wang X, Wu T, Zhang J, Guo G, He X, Pei Z, Liu Z, Liu CF, Ross CA, Smith WW: Transmembrane Protein 230 Mediates a Poly(ADP-ribose) Polymerase-1-Linked Apoptosis. Frontiers in aging neuroscience 2020, $12: 235$.

6. Wang X, Whelan E, Liu Z, Liu CF, Smith WW: Controversy of TMEM230 Associated with Parkinson's Disease. Neuroscience 2021, 453:280-286.

7. Nam D, Kim H, Choi DJ, Bae YH, Lee BD, Son I, Seol W: Characterization of Parkinson's disease-related pathogenic TMEM230 mutants. Animal cells and systems 2018, 22(2):140-147.

8. Williams ET, Chen X, Moore DJ: VPS35, the Retromer Complex and Parkinson's Disease. Journal of Parkinson's disease 2017, 7(2):219-233.

9. Vilariño-Güell C, Wider C, Ross OA, Dachsel JC, Kachergus JM, Lincoln SJ, Soto-Ortolaza Al, Cobb SA, Wilhoite GJ, Bacon JA et al: VPS35 mutations in Parkinson disease. American journal of human genetics 2011, 89(1):162-167.

10. Zimprich A, Benet-Pagès A, Struhal W, Graf E, Eck SH, Offman MN, Haubenberger D, Spielberger S, Schulte EC, Lichtner $P$ et al: A mutation in VPS35, encoding a subunit of the retromer complex, causes late-onset Parkinson disease. American journal of human genetics 2011, 89(1):168-175. 
11. McGough IJ, Steinberg F, Jia D, Barbuti PA, McMillan KJ, Heesom KJ, Whone AL, Caldwell MA, Billadeau DD, Rosen MK et al: Retromer binding to FAM21 and the WASH complex is perturbed by the Parkinson disease-linked VPS35(D620N) mutation. Current biology : CB 2014, 24(14):1670-1676.

12. Zavodszky E, Seaman MN, Moreau K, Jimenez-Sanchez M, Breusegem SY, Harbour ME, Rubinsztein DC: Mutation in VPS35 associated with Parkinson's disease impairs WASH complex association and inhibits autophagy. Nature communications 2014, 5:3828.

13. Liu Z, Wang X, Yu Y, Li X, Wang T, Jiang H, Ren Q, Jiao Y, Sawa A, Moran T et al: A Drosophila model for LRRK2-linked parkinsonism. Proceedings of the National Academy of Sciences of the United States of America 2008, 105(7):2693-2698.

14. Manjila SB, Hasan G: Flight and Climbing Assay for Assessing Motor Functions in Drosophila. Bioprotocol 2018, 8(5):e2742.

15. Hosamani R, Ramesh SR, Muralidhara: Attenuation of rotenone-induced mitochondrial oxidative damage and neurotoxicty in Drosophila melanogaster supplemented with creatine. Neurochemical research 2010, 35(9):1402-1412.

16. Simon DK, Tanner CM, Brundin P: Parkinson Disease Epidemiology, Pathology, Genetics, and Pathophysiology. Clinics in geriatric medicine 2020, 36(1):1-12.

17. Zhu Y, Zhang J, Zeng Y: Overview of tyrosine hydroxylase in Parkinson's disease. CNS \& neurological disorders drug targets 2012, 11(4):350-358.

18. Guo Q, Wang B, Wang X, Smith WW, Zhu Y, Liu Z: Activation of Nrf2 in Astrocytes Suppressed PD-Like Phenotypes via Antioxidant and Autophagy Pathways in Rat and Drosophila Models. Cells 2021, 10(8).

19. Puspita L, Chung SY, Shim JW: Oxidative stress and cellular pathologies in Parkinson's disease. Molecular brain 2017, 10(1):53.

20. Klegeris A, Pelech S, Giasson BI, Maguire J, Zhang H, McGeer EG, McGeer PL: Alpha-synuclein activates stress signaling protein kinases in THP-1 cells and microglia. Neurobiology of aging 2008 , 29(5):739-752.

21. Yang D, Thomas JM, Li T, Lee Y, Liu Z, Smith WW: The Drosophila hep pathway mediates Lrrk2induced neurodegeneration. Biochemistry and cell biology = Biochimie et biologie cellulaire 2018, 96(4):441-449.

22. Follett J, Norwood SJ, Hamilton NA, Mohan M, Kovtun O, Tay S, Zhe Y, Wood SA, Mellick GD, Silburn PA et al: The Vps35 D620N mutation linked to Parkinson's disease disrupts the cargo sorting function of retromer. Traffic (Copenhagen, Denmark) 2014, 15(2):230-244. 
23. Deas E, Cremades N, Angelova PR, Ludtmann MH, Yao Z, Chen S, Horrocks MH, Banushi B, Little D, Devine MJ et al: Alpha-Synuclein Oligomers Interact with Metal Ions to Induce Oxidative Stress and Neuronal Death in Parkinson's Disease. Antioxidants \& redox signaling 2016, 24(7):376-391.

24. Russo I, Kaganovich A, Ding J, Landeck N, Mamais A, Varanita T, Biosa A, Tessari I, Bubacco L, Greggio $\mathrm{E}$ et al: Transcriptome analysis of LRRK2 knock-out microglia cells reveals alterations of inflammatory- and oxidative stress-related pathways upon treatment with a-synuclein fibrils. Neurobiology of disease 2019, 129:67-78.

25. Kim EK, Choi EJ: Compromised MAPK signaling in human diseases: an update. Archives of toxicology 2015, 89(6):867-882.

26. Son Y, Kim S, Chung HT, Pae HO: Reactive oxygen species in the activation of MAP kinases. Methods in enzymology 2013, 528:27-48.

\section{Figures}

A
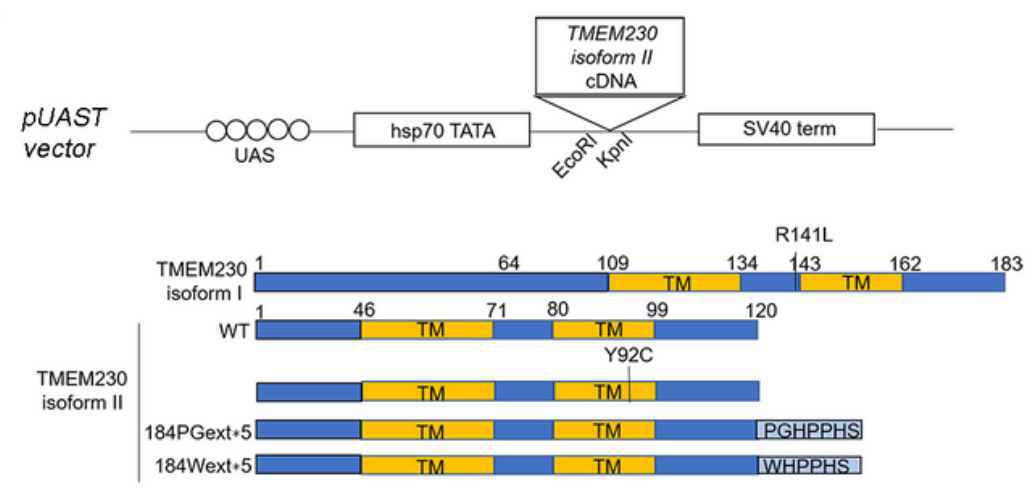

C

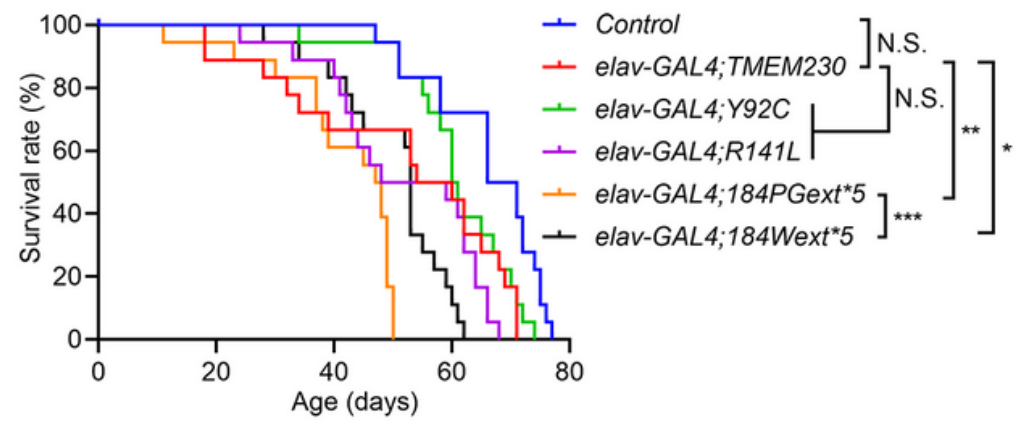

B

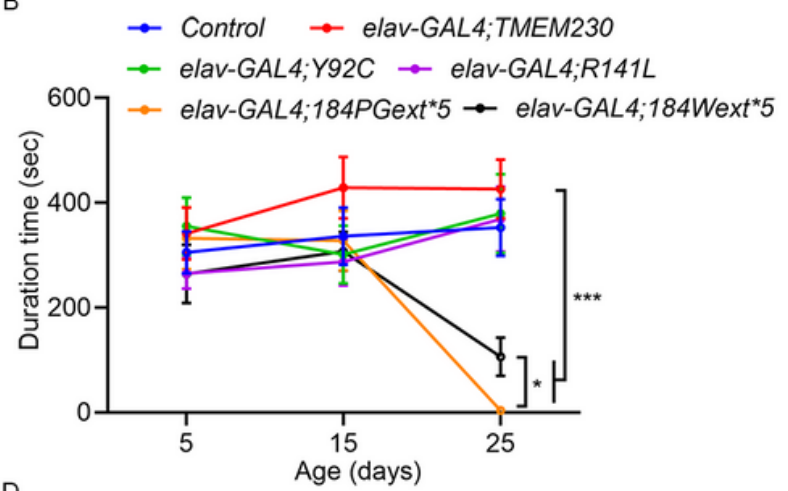

D

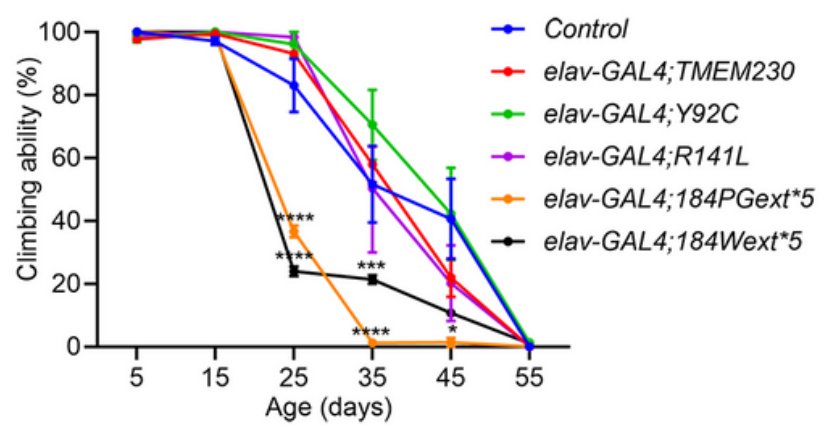

Figure 1

Expression of TMEM230 184PGext*5 and 184Wext*5 in neurons induces locomotor deficits and shortens the fly lifespan. A. Vector for UAS-TMEM230 (WT, R141L, Y92C, 184PGext*5 and 184Wext*5) gene expression is indicated upper in A, TMEM230 structure and the tested mutants in isoforms 1 and 2, the locations of two putative transmembrane domains (TM), R141L, Y92C, 184PGext*5 (PGHPPHS) and 184Wext*5(WHPPHS) are indicated below in A; B. flight assays. Elav-184PGext*5(orange) and elav- 
184Wext*5(black) showed flight deficit as compared to elav-WT flies (red); C. lifespan assays. Elav184PGext $\star 5$ and elav-184Wext*5 shortened lifespan (by log-rank test); D. climbing assays. Elav184PGext ${ }^{\star} 5$ and elav-184Wext ${ }^{\star} 5$ displayed locomotor impairment. One-way ANOVA, Turkey's test with

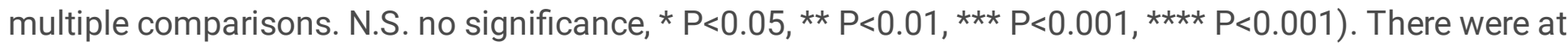
least 60 flies in each experimental group.

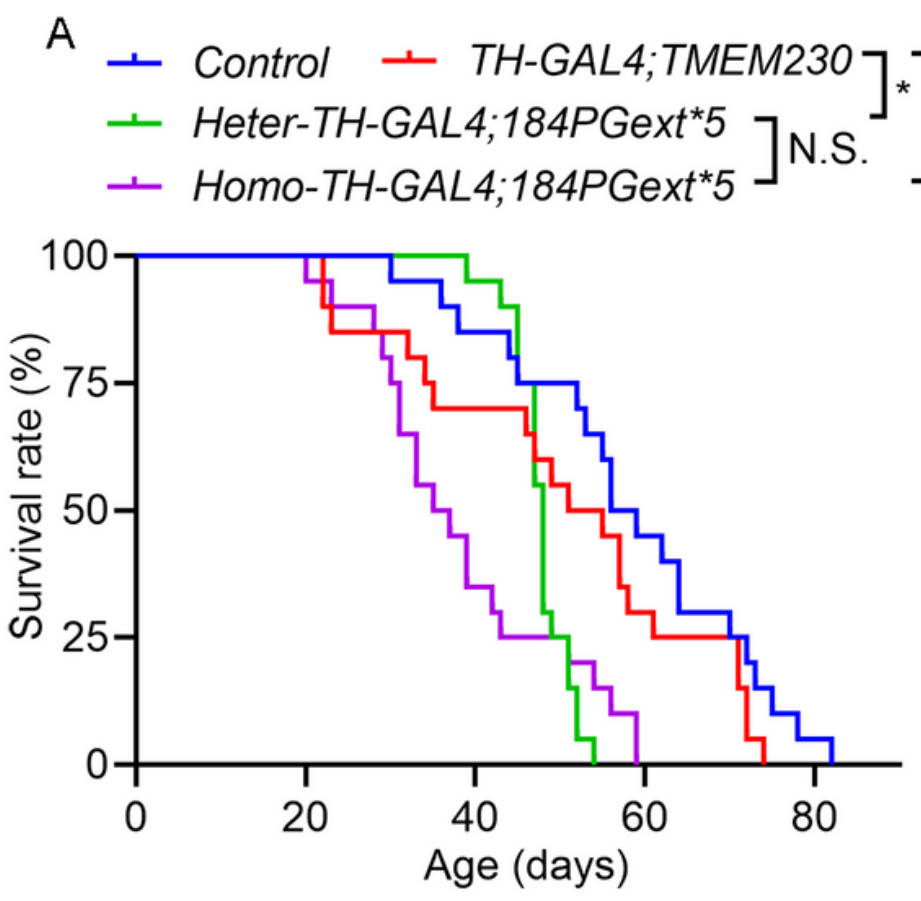

C

$\rightarrow$ Control $\rightarrow-$ TH-GAL4;TMEM230

$\Rightarrow$ Heter-TH-GAL4;184PGext*5

$=$ Homo-TH-GAL4;184PGext*5

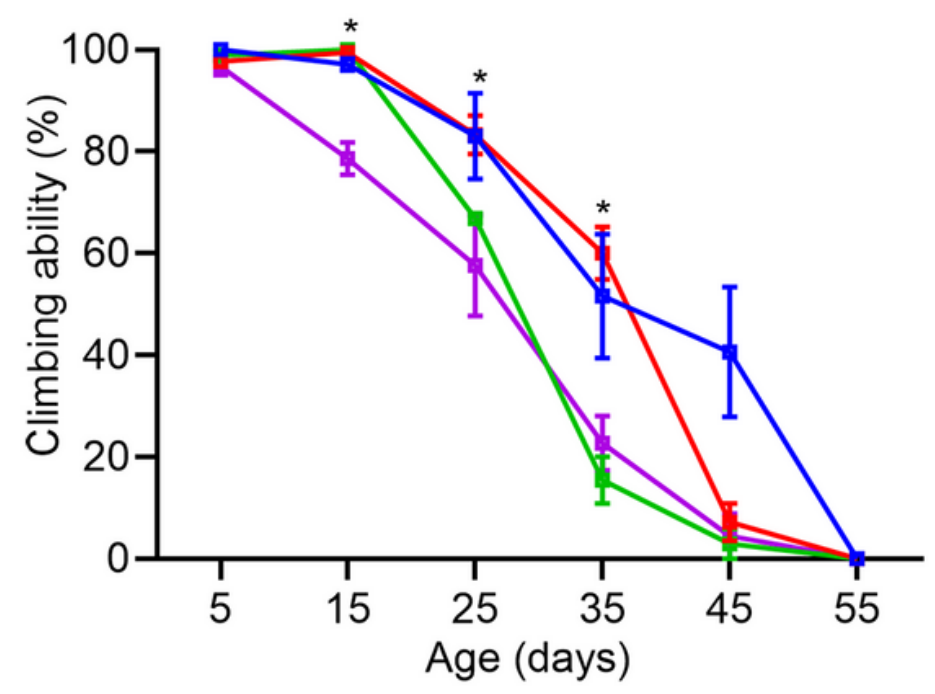

B

- Control a TH-GAL4;TMEM230

- Heter-TH-GAL4;184PGext*5

- Homo-TH-GAL4;184PGext ${ }^{* 5}$
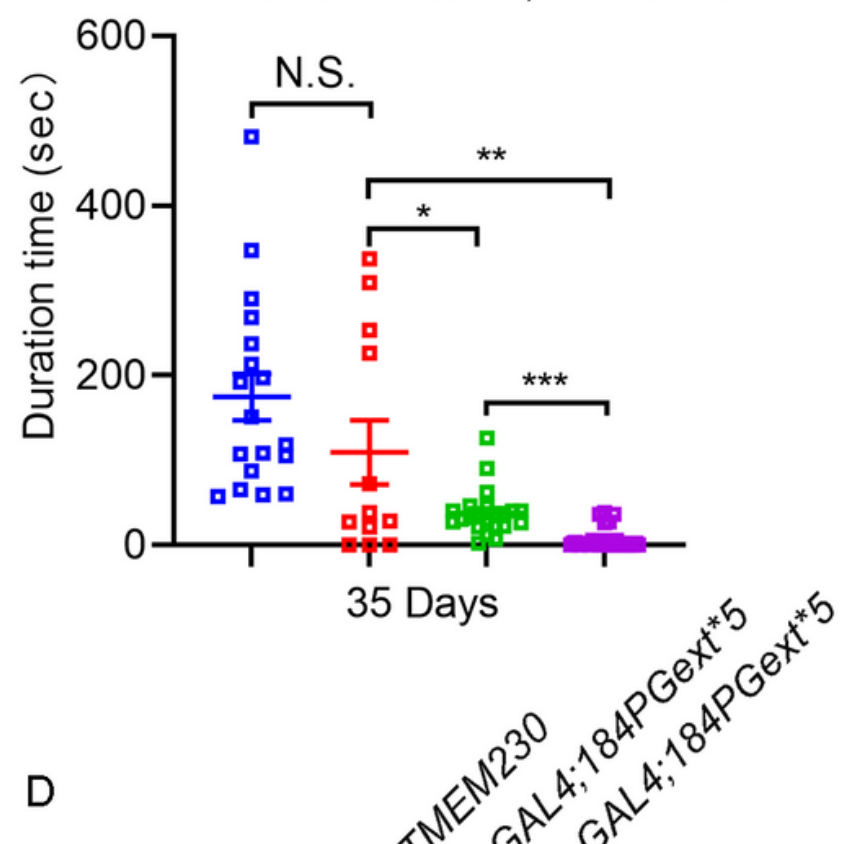
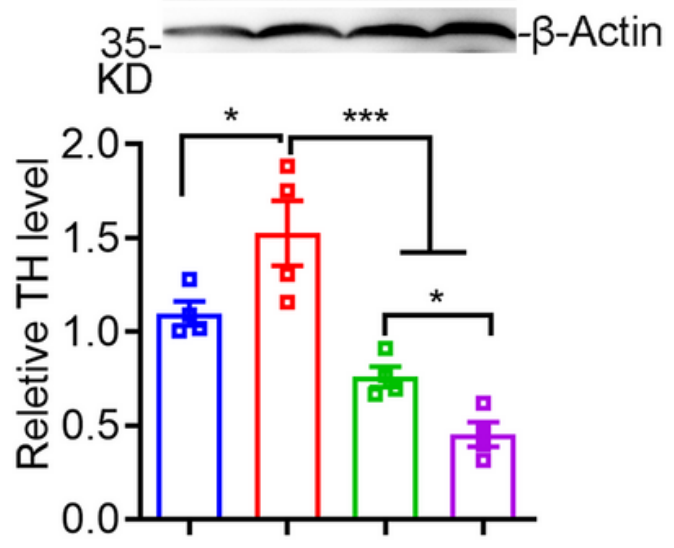

Figure 2 
Expression of TMEM230 184PGext*5 in DNs evokes locomotor deficits. A. Lifespan assays. THGAL4;184PGext*5 (green) shortened lifespan as compared to TH-GAL4; WT flies (red). Log-rank test was used to analyse the data. B. Flight assays. TH-GAL4;184PGext $\star 5$ flies displayed the impaired flight ability at the age of 35 days; C. Climbing assays. TH-GAL4;184PGext*5 displayed age-related locomotor decline; D. TH-GAL4;184PGext ${ }^{\star} 5$ decreased TH protein level in brains. The expression of TH in fly brains were measured using western blot analysis with an-TH antibody. $\beta$-actin was used as loading control. The quantity of TH proteins was normalized to $\beta$-actin. One-way ANOVA, followed by post Turkey's test for multiple group comparison ${ }^{*} \mathrm{P}<0.05$, $* * \mathrm{P}<0.01$, ${ }^{* \star} \mathrm{P}<0.001$. There were at least 60 flies in each experimental group.

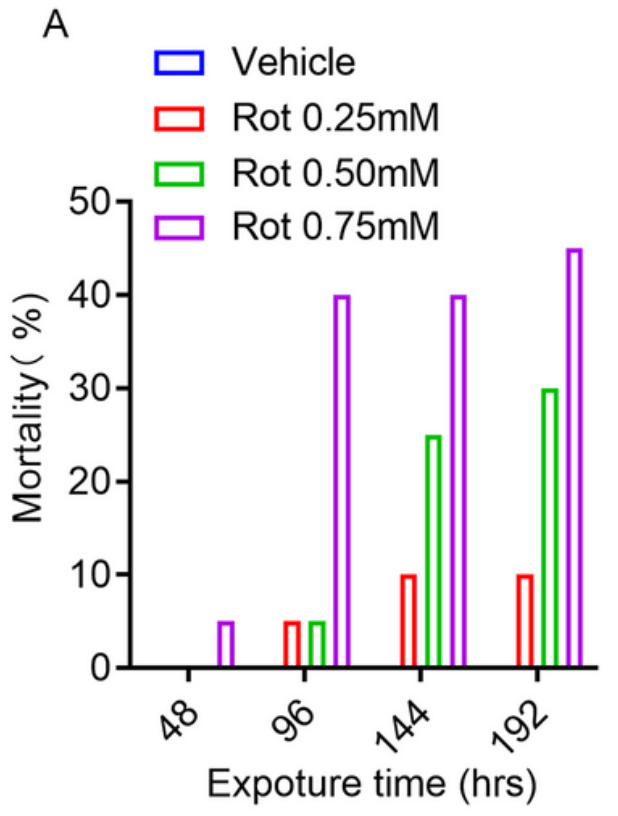

B

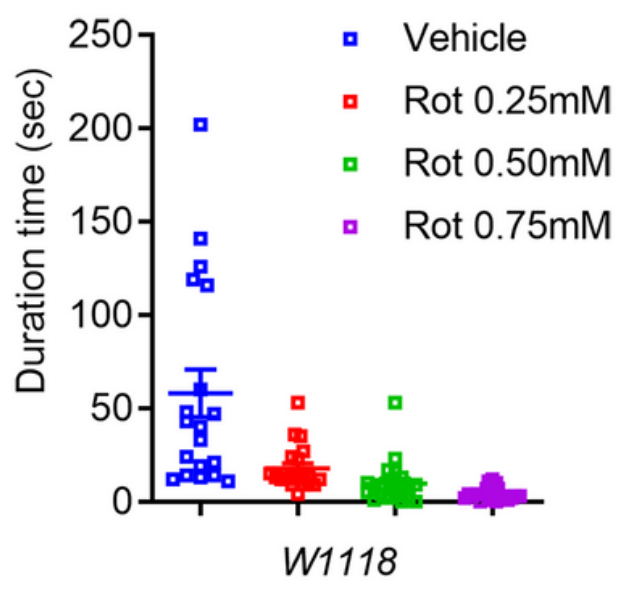

D

TH-GAL4;TMEM230

TH-GAL4;184PGext*5

C
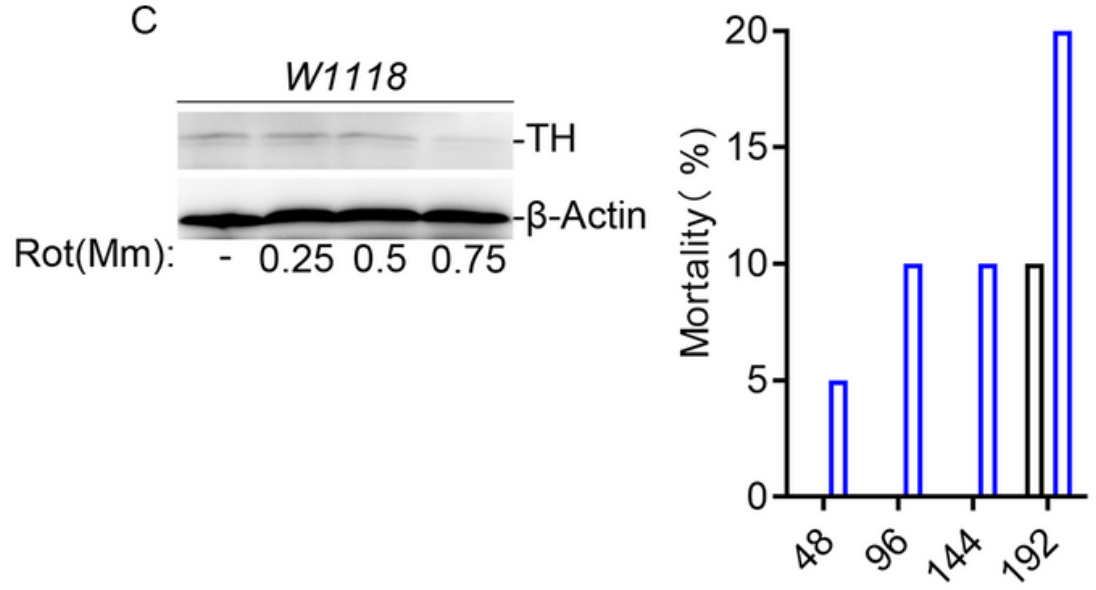

(hrs)
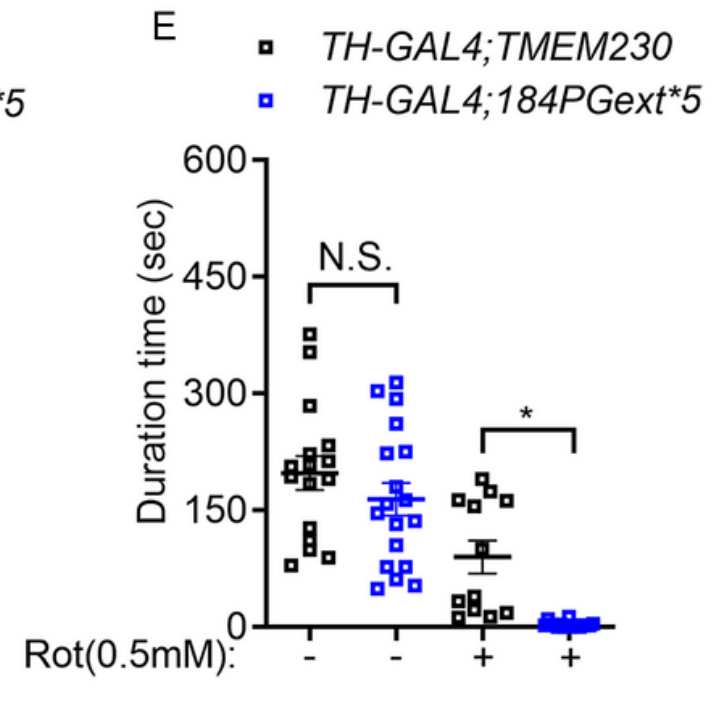

$\operatorname{Rot}(0.25 \mathrm{mM})$

Figure 3 
Expression of TMEM230 184PGext*5 in DNs enhances Rot-induced toxicity in flies. A. Mortality of adult flies exposed to Rotenone $(0.25,0.5$ and $0.75 \mathrm{mM}$ ) was calculated; $B$. Adult flies exposed to Rotenone were subjected to flight assays; $\mathrm{C}$. The expression of $\mathrm{TH}$ in fly brains after exposed to Rotenone was measure by anti-TH immunoblotting assays. Anti- $\beta$-actin immunoblotting was used as protein loading control; D. Mortality of TH-GAL4;184PGext 5 flies increased after exposing Rotenone at $0.25 \mathrm{mM}$ concentration; E. Expression of TH-GAL4;184PGext*5 exacerbated Rotenone-induced locomotor impairment; N.S. no significance, * $\mathrm{P}<0.05$, One-way ANOVA, Turkey's test with multiple comparisons. There were at least 60 flies in each experimental group.

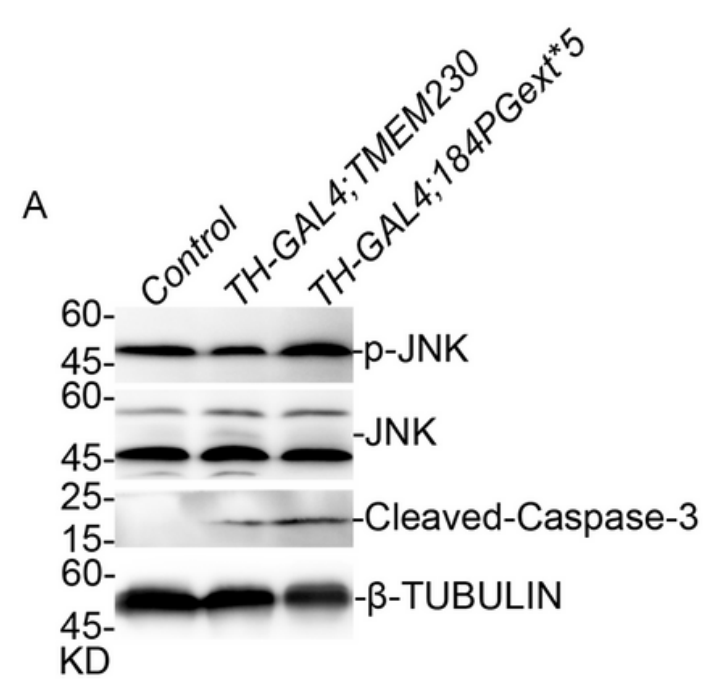

B

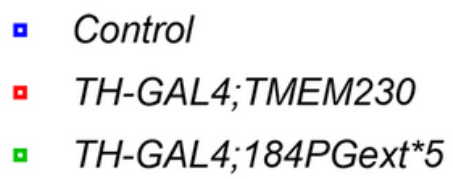

- Control - TH-GAL4;184PGext*5

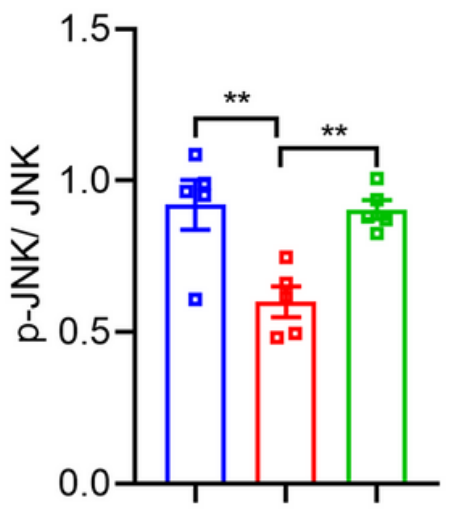

C

- Control

- TH-GAL4;TMEM230

- TH-GAL4;184PGext ${ }^{*} 5$

\section{Figure 4}

TMEM230 184PGext ${ }^{\star}$ induces JNK phosphorylation and caspase- 3 cleavage. A. TH-GAL4;184PGext*5 induced JNK/caspase-3 activation compared with WT-TMEM230 transgenic flies. The expression of $p$ JNK, JNK and cleaved-Caspase-3 in fly brain tissue from adult flies were measured by western blot analysis with antibodies against $\mathrm{p}$-JNK, JNK, cleaved-Caspase- 3 antibody and $\beta$-tubulin; B. Quantification of p-JNK, JNK in A; C. Quantification of cleaved-Caspase-3 in A; One-way ANOVA, Turkey's test with multiple comparisons. N.S. means no significance, ${ }^{*} P<0.05$, $* * P<0.01$.

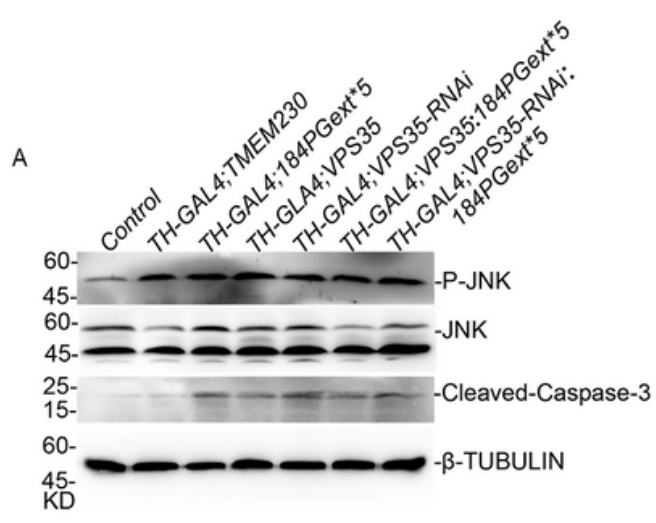

B

- Control - TH-GAL4;TMEM230

- TH-GAL4;184PGext*5 a TH-GAL4;VPS35

- TH-GAL4;VPS35-RNAi

- TH-GAL4;VPS35:184PGext*5

- TH-GAL4;VPS35-RNAi:184PGext*5

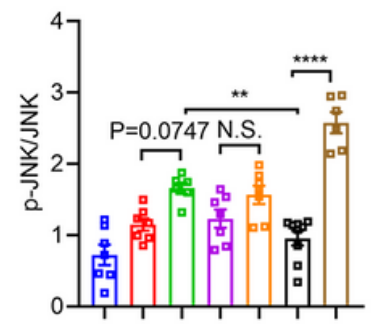

C

- Control a TH-GAL4;TMEM230

- TH-GAL4;184PGext ${ }^{\star} 5$ a TH-GAL4;VPS35

- TH-GAL4;VPS35-RNAi

- TH-GAL4;VPS35:184PGext*5

- TH-GAL4;VPS35-RNAi:184PGext ${ }^{* 5}$

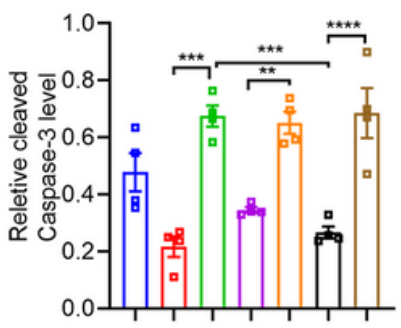




\section{Figure 5}

Expression of VPS35 in DNs reduces TMEM230-184PGext*5-induced JNK phosphorylation and caspase3 activation. A. The levels of p-JNK, JNK, cleaved-Caspase-3 were measured by western blot analysis using antibodies against p-JNK, JNK, cleaved-Caspase-3 and $\beta$-tubulin; B. TH-GAL4; VPS35 decreased phosphorylation level of JNK in TH-GAL4; VPS35:184PGext 5 flies. p-JNK was measured by western blot analysis; C. TH-GAL4; VPS35 decreased Caspase-3 cleavage in TH-GAL4; VPS35: 184PGext^5 flies.

Cleaved-caspase- 3 was measured by western blot analysis. The quantity of proteins was normalized to $\beta-$ tubulin; One-way ANOVA, Turkey's test with multiple comparisons. N.S. means no significance, ${ }^{* \star} P<0.01$, $\star * \star P<0.001 * \star * \star P<0.0001$. 


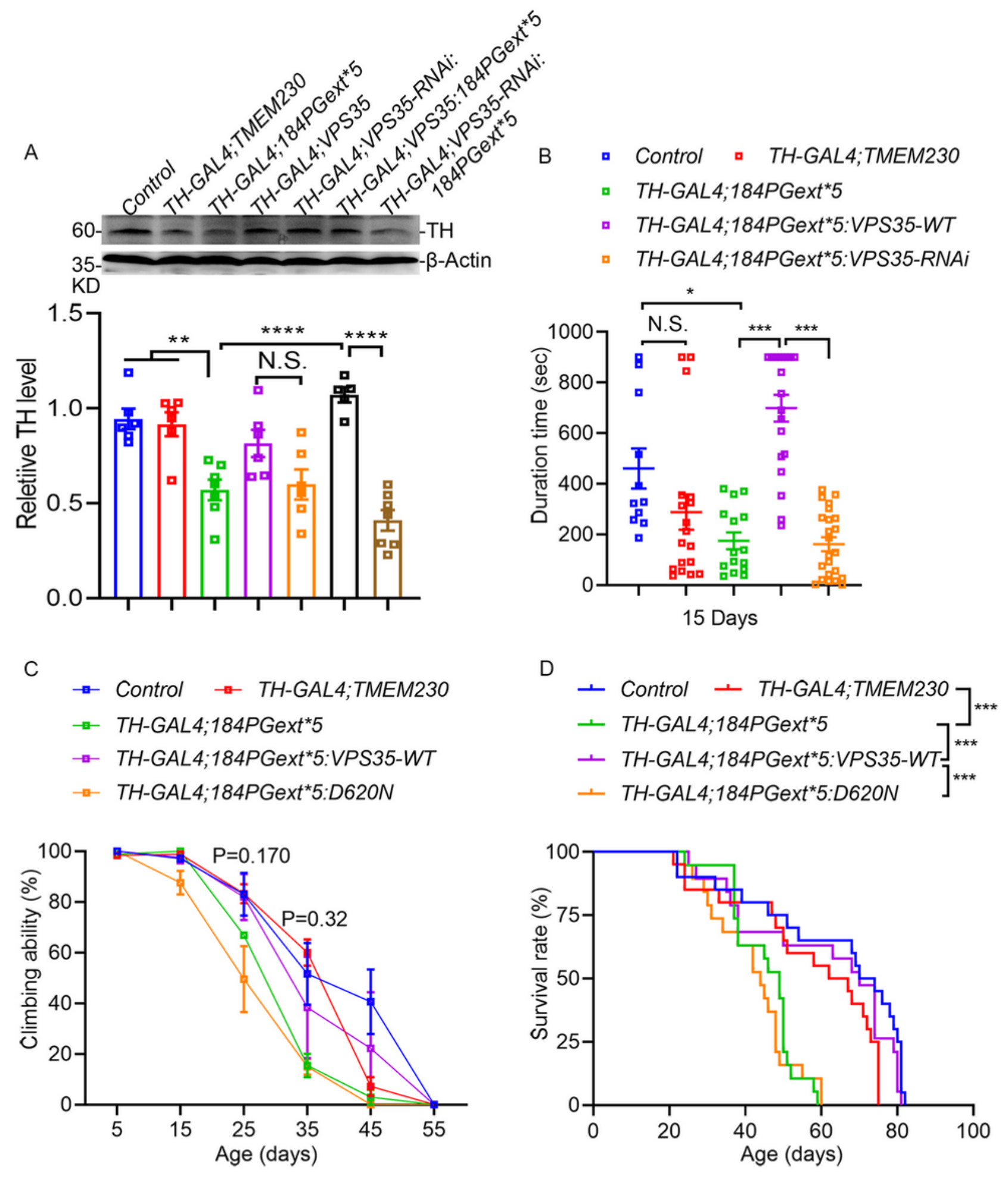

Figure 6

Expression of VPS35 in DNs attenuates TMEM230-184PGext*5-induced PD-like phenotypes in flies. A. TH-GAL4; VPS35 increased TH in TH-GAL4; VPS35: 184PGext`5 flies. TH was measured by western blot analysis. The quantity of TH proteins was normalized to $\beta$-actin; B. Flight assays. TH-GAL4; VPS35 extended the duration time of flight in TH-GAL4; VPS35:184PGext*5 flies; C. Climbing assays. TH-GAL4; VPS35 improved 184PGext^5-induced locomotor impairment in TH-GAL4; VPS35: 184PGext^5 flies; D. 
Survival assays. TH-GAL4; VPS35 extends the survival time of TH-GAL4; VPS35: 184PGext^5 flies.

Survival curves of indicated genotypes are shown and the statistical significance was analysed by logrank test. Data from flight assays and climbing assays were analysed by One-way ANOVA, Turkey's test

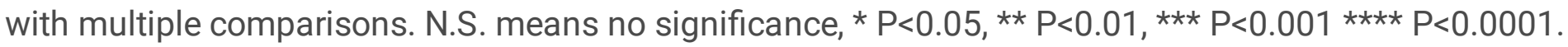

